CONTRIBUTED PAPERS 


\title{
SIXA: THE SOLID STATE SPECTROMETER ARRAY ONBOARD SPECTRUM-X-GAMMA
}

\author{
O. VILHU \\ Observatory and Astrophysics Laboratory, University of Helsinki, \\ SF-00130 Helsinki, Finland \\ H. SIPIL $\ddot{A}$ \\ Outokumpu Electronics, Box 85, SF-02201 Espoo, Finland \\ V.J. KÄMÄRÄINEN \\ Outokumpu Electronics, Box 85, SF-02201 Espoo, Finland \\ I. TAYLOR \\ Princeton Gamma-Tech Inch. PGT, Princeton, NJ, USA \\ E. LAEGSGAARD \\ Institute of Physics, Univ. of Aarhus, Denmark \\ G. LEPPELMEIER \\ Technical Research Center, Instrument Lab., SF-02150 Espoo, Finland \\ and \\ H.W.SCHNOPPER \\ Danish Space Research Institute, Lundtoftevej 7, DK-2800 Lyngby, Denmark
}

\begin{abstract}
The SPECTRUM - X-GAMMA mission is being developed by the Space Research Institute (IKI), USSR, together with many other countries and is scheduled for launch in 1993 ( Sunyaev,1990; Schnopper,1990). Mission objectives include broad and narrow band imaging spectroscopy over a wide range of energies from the EUV through gamma rays, with an emphasis on studying galactic and extragalactic X-ray sources. The Danish Space Research Institute (DSRI) and IKI will provide two thin-foil X-ray telescopes (SODART), each with an aperture of $60 \mathrm{~cm}$ and focal length of $8 \mathrm{~m}$. They are designed to have a half-power width of less than 2 arc minutes and will have collecting areas of 1700 , 1200 and $100 \mathrm{~cm}^{2}$ at 1,8 and $20 \mathrm{keV}$, respectively. Images and polarization will be recorded by position-sensitive proportional counters. Moderate resolution spectroscopy will be done by the segmented solid state detector SIXA (SIlicon X-ray Array), designed and to be constructed by a consortium in Finland, Denmark and USSR. Finland will have the main responsibility in financing and delivering the detector. The Institute of Electromechanics in Moscow will provide its passive cooling system $(110 \mathrm{~K})$. The detector will consist of 19 segments $(\mathrm{Si}(\mathrm{Li}))$, each with a diameter of about $8 \mathrm{~mm}$. The spectral resolution of $160 \mathrm{eV}$ (at $6 \mathrm{keV}$ ), combined with the large collecting area, provide good opportunities for time-resolved iron line spectroscopy ( 6-8 keV). The potential observing program includes stellar coronae, cataclysmic variables and X-ray binaries, accretion discs and coronae of neutron stars and black hole candidates, supernova-remnants, active galactic nuclei, clusters of galaxies and the diffuse cosmic X-ray background. We demonstrate the instrument's power through some astrophysical simulations.
\end{abstract}

Y. Kondo (ed.), Observatories in Earth Orbit and Beyond, 433-438.

(C) 1990 Kluwer Academic Publishers. Printed in The Netherlands. 
TABLE I

The SIXA-detector parameters.

Diameter of active area

FOV

Number of pixels

Pixel diameter

Number of energy channels

Width of each channel

Energy range

Efficiency

Depletion depth

Energy resolution (FWHM)

Operation temperature

Power consumption/pixel
$4 \mathrm{~cm}$

15-18 arc min

6-8 $\mathrm{mm}$

1000

$20 \mathrm{eV}$

$0.5-30 \mathrm{keV}$

over $0.85(2-20 \mathrm{keV})$

$3 \mathrm{~mm}$

$140 \mathrm{eV}$ at $2 \mathrm{keV}$

$160 \mathrm{eV}$ at $6 \mathrm{keV}$

$250 \mathrm{eV}$ at $20 \mathrm{keV}$

$110 \mathrm{~K}$ $13 \mathrm{~mW}$

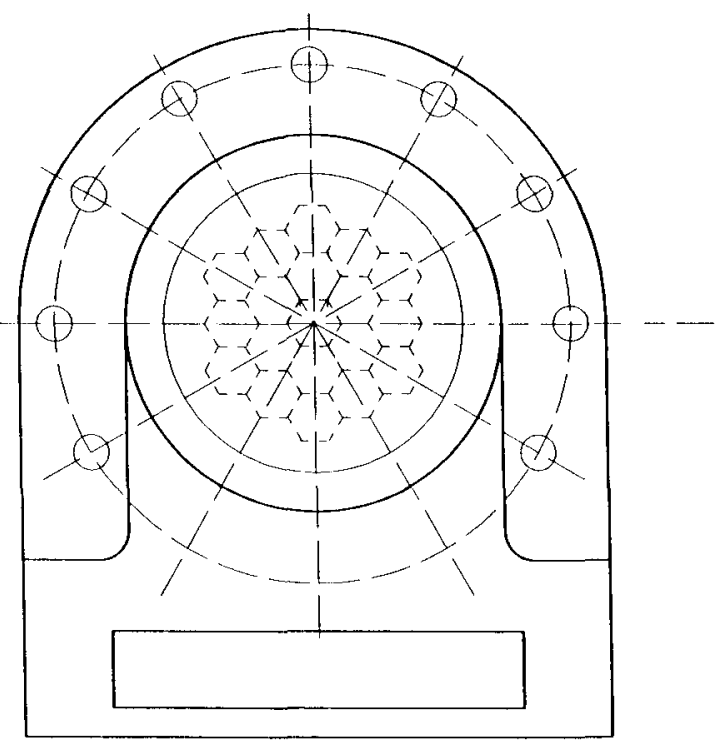

Fig. 1. The SIXA detector unit. 
SIXA-BEAM ON G109.1-1.0 (SN-REMNANT)

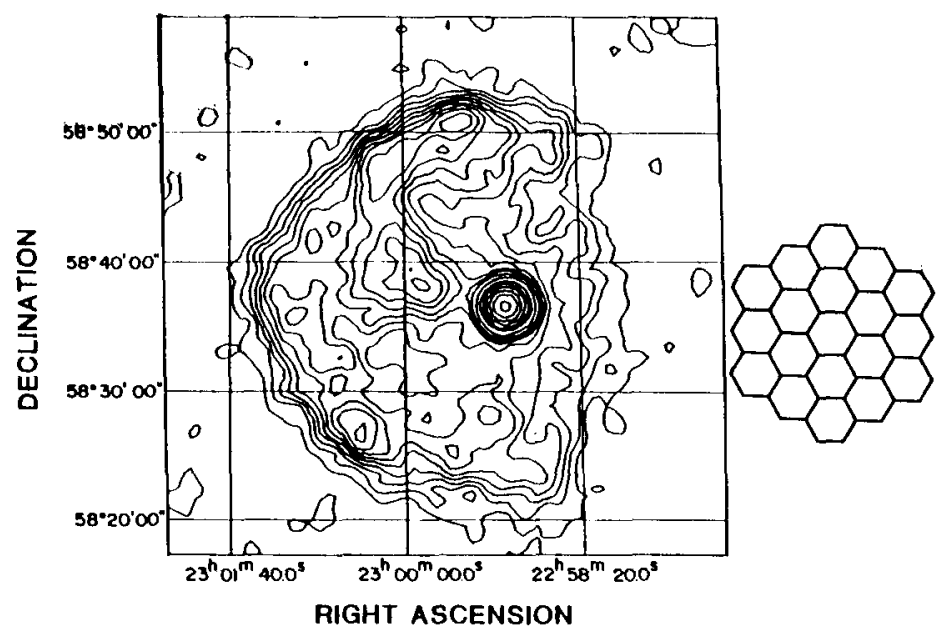

Fig. 2. The SIXA and the Supernova remnant G109.1-1.0.

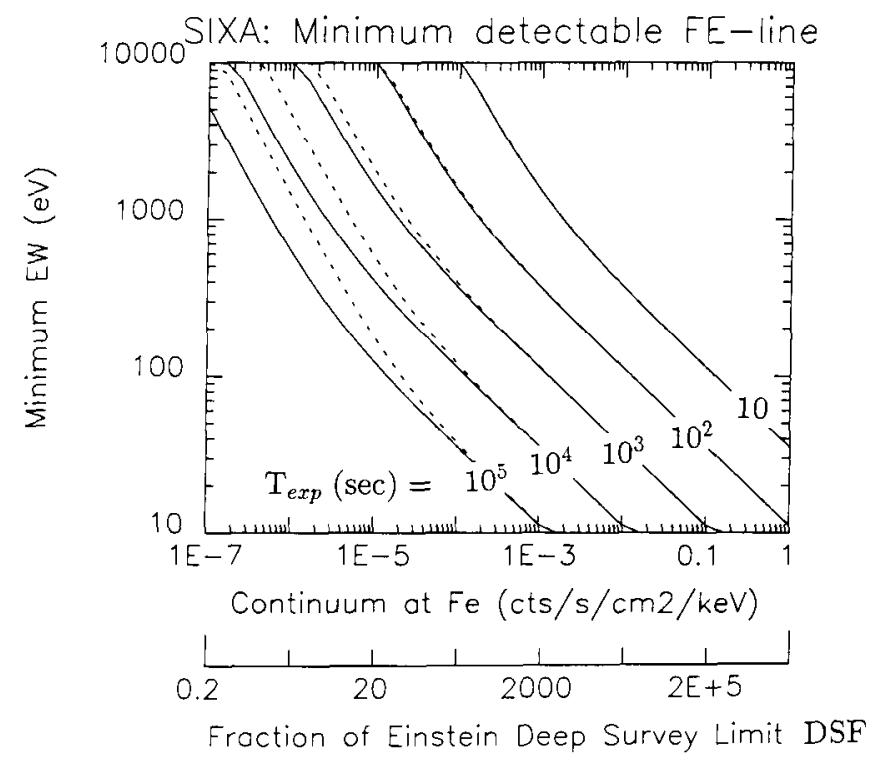

Fig. 3. Exposure times for the iron line detection. 


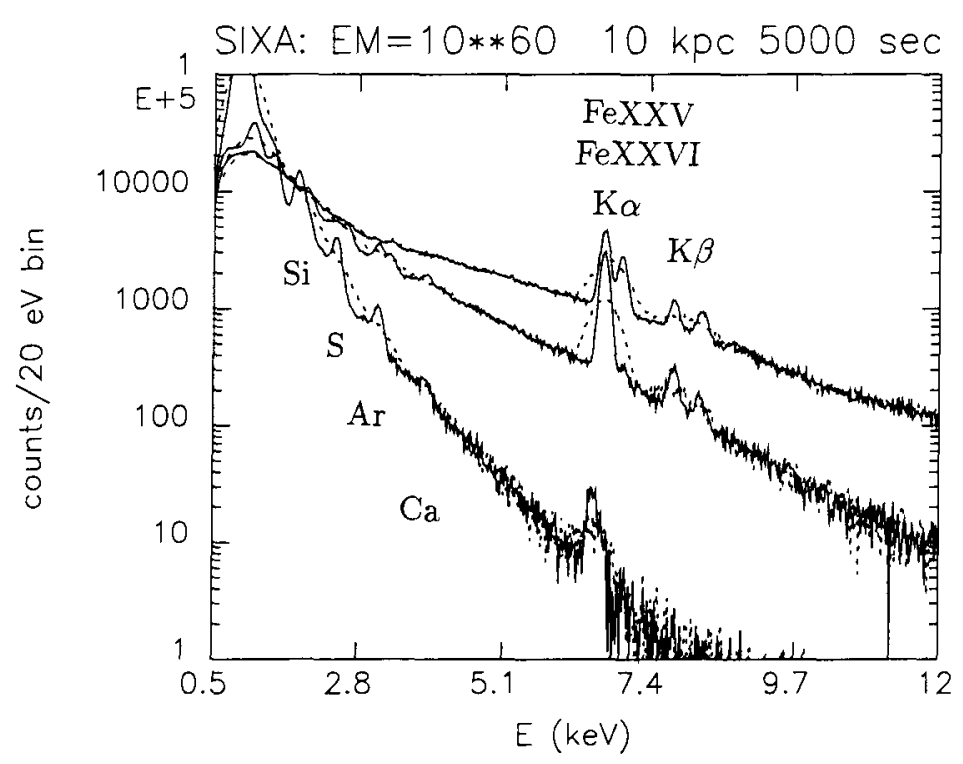

Fig. 4. Spectra of hot thin plasmas as seen by SIXA.

\section{The Detector}

The significant improvement in energy resolution obtained from solid state devices (as compared with the gas detectors) more than compensates the requirement that the device should be cooled. Solid state X-ray detectors are made of lithium-drifted silicon ( $\mathrm{Si}(\mathrm{Li})$ ). The primary difference from a gas detector is that, although much less energy is required to liberate an electron within the silicon, there is no equivalent to gas amplification. The primary charge must be detected by extremely low noise. Cooled preamplifiers can contribute $100 \mathrm{eV}$ to the FWHM and this is the limiting resolution for $\mathrm{X}$-rays below $2 \mathrm{keV}$. At $6 \mathrm{keV}$, a resolution $150 \mathrm{eV}(\mathrm{R}=40)$ can be reached.

The deep orbit makes it possible to use a radiative cooler to bring the detector system to the proper operating temperature $(110 \mathrm{~K})$. The optimal pixel-size of the detector matrix is of the order of the point spread function of a point source as seen by the SODART-telescope, and a pixel size of 6-8 $\mathrm{mm}$ was chosen. A larger pixel size would degrade the energy resolution and also increase the background. The number of pixels is limited by the minimum power required by the preamplifier and the heat leaks between the detector assembly and the heat radiator. The SIXAinstrument will have 19 hexaconal pixels (independent segments), giving a total field of view of about 15-20 arc minutes (see Figs. 1 and 2). Especially when observing weak sources and crowded regions, a sufficient number of pixels is important for background rejection and to retain some imaging capability. Within the expected accuracy (a few arc minutes) of the satellite pointing system, nineteen segments serves a safe solution for bright point source observations, as well. 
Table I gives the basic parameters of the detector-array and Fig. 1 shows schematically the detector unit. In Fig. 2 the SIXA-beam is overlayed on the supernova remnant G109.1-1.0 (including the central X-ray pulsar 1E2259+586, Fahlman and Gregory, 1981).

\section{Spectroscopy and Timing of X-Ray Sources}

The high sensitivity, broad energy range and spectral resolution of the SIXAdetector will provide high quality, time-resolved X-ray spectra from a large sample of astronomical objects (for a good review see the XMM science report, ESA SP1097). The energy range contains the K-shell transitions of oxygen, neon, magnesium, aluminium, silicon, sulphur and calcium, as well as L- and K-shell transitions of iron. Of particular interest is, due to its high abundance, iron at temperatures $10^{7}-10^{8} \mathrm{~K}$. Detailed analysis of these spectral features will permit the determination of the physical characteristics of the emitting region and its surrounding environment. The iron $\mathrm{K} \alpha$ line has the energy between $6.4-7.0 \mathrm{keV}$, depending on the ionization state of the plasma and the excitation mechanism. The equivalent widths of this line in X-ray binaries and active galactic nuclei (AGN) are typically 50-200 eV, as observed by the previous missions (EXOSAT and GINGA).

Fig. 3 shows the minimum detectable iron line with the SIXA-detector (at the $5 \sigma$ level), computed as a function of the exposure time and continuum level. The strongest galactic X-ray sources have continuum levels $0.1 \mathrm{cts} / \mathrm{s} / \mathrm{cm}^{2} / \mathrm{keV}$, and an integration time of 10 seconds can give the detection. The iron line in sources as weak as 20 DSF (DSF=The Einstein Deep Survey Limit) can be detected in one day of observation. An example would be NGC4151 at a distance of $100 \mathrm{Mpc}$ (the actual distance is $10 \mathrm{Mpc}$ ).

Compact X-ray binaries are accretion-powered radiation sources where a normal non-degenerate star transfers mass to a compact star (white dwarf, neutron star or black hole). Spectroscopy with sufficient time resolution would give valuable new information about the accretion process and ultimately the compact star itself. The line profiles are affected by the following broadening mechanisms (Kallmann and White, 1989):

- blending of different line components (see Fig. 4: $\mathrm{T}=10^{8} \mathrm{~K}, 10^{7.5} \mathrm{~K}$ and $10^{7} \mathrm{~K}$ (lowest), dashed curves: FWHM $=0.5 \mathrm{keV}$ ).

- thermal broadening which is very small even for the highest temperatures, as compared with the SIXA-resolution $(160 \mathrm{eV})$.

- Compton broadening $\delta \mathrm{E}=\mathrm{E}(4 \mathrm{kT}-\mathrm{E}) /\left(\mathrm{m}_{e} \mathrm{c}^{2}\right)$, which is a result of the energy shift due to Compton scattering, and can reach $1 \mathrm{keV}$ for a Thompson depth of 3 .

- Doppler broadening due to bulk motions of the plasma in the vicinity of a collapsed object. In the case of a Keplerian motion $\delta E=\left(r / 50 r_{s}\right)^{-1 / 2} \mathrm{keV}$, where $\mathrm{r}_{s}$ is the $\mathrm{Schwarzschild}$ radius $2 G M / c^{2}$. In addition, general relativistic effects might be present in line profiles (Stella, 1989).

With the SIXA-detector, accretion discs and their coronae can be studied in detail. Accumulating spectra from different X-ray pulse- or burst-phases, the physics of these short-lived phenomena can also be investigated. In AGN's the line pro- 
file studies are equally important. The spectral resolution of SIXA at the iron line $(E / \delta E=40)$ is very suitable for these kinds of observations.

\section{Observing Modes}

Three observing modes will be used: S.E.C.-mode (single event characterization), E-mode (energy-spectrum) and T-mode (timing in pass-bands). The modes can be run in parallel or separately. In the S.E.C.-mode every event is stored (i.e. the photon energy (energy channel), the photon arrival time and the id of the segment). The arrival time can be determined with accuracy of $1 \mathrm{msec}$. The detector itself can separate events within a few microseconds, and this is used (by anticoincidence) for background rejection.

In the E-mode the integration time is specified by the user. Within this interval the counts in each of the 1000 channels are summed. The optimal integration time depends on the source strength. At least several thousand counts have to be recorded before any useful spectrum can be stored. For point-source observations this mode is not very memory-economic if spectra from all 19 segments are stored. However, in that case a very good background elimination can be made.

In the T-mode the user selects the integration time (time-bin) and the energy bands within which the counts are summed. Typically, the energy bands could be $0.5-2 \mathrm{keV}$ (soft), 2-10 keV (medium) , 10-20 keV (hard) and 6-7.5 keV (the iron-line).

The memory available will put constraints on the observing modes. If 40 Mbytes of memory will be available, then in the S.E.C.-mode all events from a 100 counts/sec-source can be stored in a $10^{5} \mathrm{sec}$ observation. Alternatively, 1000 spectra (from all 19 segments) in the E-mode, or $1.310^{6}$ time bins in the T-mode with 4 windows ( $\delta t=80 \mathrm{msec}$ ), can be stored. In addition to these 3 main observing modes, specific modes will be developed to study very short timescale (msec size) and iron line variabilities in strong X-ray binaries.

\section{References}

Fahlman G.G. and Gregory P.C.: 1981, Nature 293, 202

Kallman,T. and White N.: 1989, Astrophys.J. 341, 955

Schnopper H.W., 1990, This volume

Stella L.: 1989, ESA SP 296, 19

Sunyaev R., 1990, This volume

XMM The Mission Science Report, 1988, ESA SP, 1097 